\title{
Association between pesticide exposure and risk of kidney cancer: a meta-analysis
}

This article was published in the following Dove Press journal:

OncoTargets and Therapy

28 June 2016

Number of times this article has been viewed

Bo Xie

Yingfang $\mathrm{Hu}$

Zhen Liang

Ben Liu

Xiangyi Zheng

Liping Xie

Department of Urology, The First Affiliated Hospital, School of Medicine, Zhejiang University, Hangzhou, Zhejiang Province, People's Republic of China
Correspondence: Liping Xie Department of Urology, The First Affiliated Hospital, School of Medicine, Zhejiang University, 79 Qingchun Road, Hangzhou 310003, Zhejiang Province, People's Republic of China

Tel +8657187236735

Fax +86 57| 87072577

Email xielp@zjuem.zju.edu.cn
Abstract: This meta-analysis aimed to evaluate the correlation between pesticide exposure and kidney cancer. We conducted a systematic search of the Cochrane Library, Embase, Web of Knowledge, and Medline (updated to March 1, 2015) to identify all relevant studies. References of the retrieved articles were also identified. Fixed- or random-effect models were used to summarize the estimates of relative risk (RR) with $95 \%$ confidence interval for the association between exposure of pesticide and risk of kidney cancer. The pooled RR estimate indicated that pesticide exposure might have an elevated risk for kidney cancer $(R R=1.10,95 \%$ confidence interval 1.01-1.19). In a subgroup analysis of high quality articles, we detected that pesticide exposure is a significant risk factor for kidney cancer in a subgroup analysis of case-control studies, (Newcastle-Ottawa Quality Assessment Scale score $>6)(\mathrm{RR}=1.31,95 \%$ confidence interval 1.12-1.51). North America studies, odds ratio studies, and studies with effect estimate adjusted for more than two confounder studies. In conclusion, pesticide exposure may be a risk factor for kidney cancer.

Keywords: pesticide exposure, kidney cancer, meta-analysis, epidemiology

\section{Introduction}

Kidney cancer is one of the most lethal malignancies in urologic neoplasms. Its incidence has increased rapidly over recent years. ${ }^{1}$ The etiology of kidney cancer is still unclear. Obesity, ${ }^{2}$ smoking, ${ }^{3}$ hypertension, antihypertensive, and heredity might lead to kidney cancer. Other risk factors are not clearly known. Their roles in severity, progression, and outcome of kidney cancer need further exploration.

Pesticides are reported to be toxic to organs. ${ }^{4}$ Both environmental and occupational exposures of pesticides could be a potential risk for cancers. ${ }^{5-7}$ Meta-analyses showed pesticide exposure was related to prostate cancer. ${ }^{6,8}$ What is worse, the metabolites of some pesticides were excreted by kidney, which might be connected with kidney cancer. Therefore, epidemiological investigations of exposures to pesticides and risk of kidney cancer were carried out. Nevertheless, the results of these findings were inconsistent.

Meta-analysis is a valuable tool for demonstrating trends which might not be apparent in a single study. Therefore, summarizing independent studies increase the confidence in the results. So, we initiated a meta-analysis of the literature to assess the effects of pesticide use on the risk of kidney cancer according to the evidence currently available.

\section{Materials and methods Systematic search strategy}

We conducted a systematic search of the Cochrane Library, Embase, Web of Knowledge, and Medline (updated to March 1, 2015) to identify all relevant studies. 
"Renal cancer OR renal cell carcinoma" AND "pesticides OR fungicides OR insecticides OR occupational exposure" were selected as keywords to identify the publications. Titles and abstracts were reviewed. If it was not clear from the abstract whether the paper contained relevant data, the full paper was assessed. The references cited in all full-text articles were also assessed for additional relevant articles.

\section{Inclusion and exclusion criterion}

Trials conducted to evaluate the potential relationship between pesticide exposure and the risk of kidney cancer were included. The included studies should include relative risk (RR), or odds ratio (OR), or standardized incidence ratio (SIR) and its 95\% confidence intervals (CIs), or it provides us with sufficient data to calculate them, no matter whether the study design is a case-control or cohort study. We excluded case series, case reports, and animal studies.

If more than one publication from the same population was available, the study with the largest number of cases was included. Trials with insufficient or overlapping data were excluded.

\section{Data extraction}

The data were extracted from the articles by including the name of the first author, publication year, region, kinds of pesticides, period of the study, follow-up time, study design, sex, intensity level, and adjusted effect estimates for all categories of pesticide exposure. Considering kidney cancer is a relatively rare disease, the absolute risk of kidney cancer is low, and the three measures of association (SIR, RR, and $\mathrm{OR}$ ) were expected to yield similar estimates of RR. ${ }^{9,10}$ All RR estimates were pooled together to maximize the comprehensiveness and statistical power of the analysis. ${ }^{11}$ Two investigators extracted the data independently. Then, a standardized form was created (Table 1).

\section{Quality assessment}

The quality of each trial was evaluated by the NewcastleOttawa Quality Assessment Scale (NOS) (http://www.ohri. ca/programs/clinical epidemiology/oxford.asp). The NOS used a "star system" to judge the quality of article by three broad perspectives: the selection of the study groups; the comparability of the groups; and the ascertainment of either the exposure or outcome of interest for case-control or cohort studies, respectively. The number of stars was calculated between 0 and 9 . Those getting scores over 7 were regarded as high-quality studies. The assessment was carried out by two authors. If there was any disagreement, a third author would reevaluate the original study.

\section{Statistical analysis}

We used RR and $95 \%$ CI to assess the relationship between pesticide exposure and risk of kidney cancer. The MantelHaenszel estimates were used and pooled under a fixed- or random-effect model when appropriate. Quantified Q test and $I^{2}$ test were used to evaluate the degree of heterogeneity across the included studies. Heterogeneity was confirmed with a significance level of $P<0.05$. Studies with an $P^{2}<25 \%$ were considered as no heterogeneity; $I^{2}=25 \%-50 \%$ as moderate heterogeneity; and $I^{2}>50 \%$ as large heterogeneity. The above mentioned analyses were performed by RevMan v.5.2. Additionally, the Egger's test and the Begg's test were used to evaluate the publication bias by STATA v.11.0 (StataCorp, College Station, Texas, USA).

\section{Results}

\section{Description of the meta-analysis}

The search algorithm yielded 785 records, of which 720 were excluded as irrelevant based on titles and abstracts. The remaining 55 studies were assessed for eligibility by full-text articles of which 44 studies were excluded with reasons, as shown in Figure 1. Finally, eleven articles were included. ${ }^{12-22}$ The details of the eligible articles are shown in Table 1. Articles including different type of pesticides, sexes, and regions were considered to be independent studies.

\section{Quality of included studies}

Rating of the quality of studies based on the NOS score is presented in Table 2. Quality scores ranged from 5 to 8 . As described above, eight articles were considered as high quality $(>6)$ and three articles as moderate. ${ }^{19,21,22}$ Rafnsson $^{19}$ and Wiklund and Dich ${ }^{22}$ did not ensure the comparability by adjusting on age or other variables. Neither Wesseling et $\mathrm{al}^{21}$ nor Wiklund and $\mathrm{Dich}^{22}$ reported the follow-up time in their articles.

\section{Pesticide exposure and kidney cancer}

Among the included studies, the pooled RR estimated indicated that pesticide exposure might have elevated the risk for kidney cancer ( $R R=1.10,95 \%$ CI 1.01-1.19, random effects). Nevertheless, a large heterogeneity was detected $\left(I^{2}=69 \%, P<0.01\right)$ (Figure 2). Egger's test and the funnel plots showed little publication bias in overall analysis (Figure 3). 


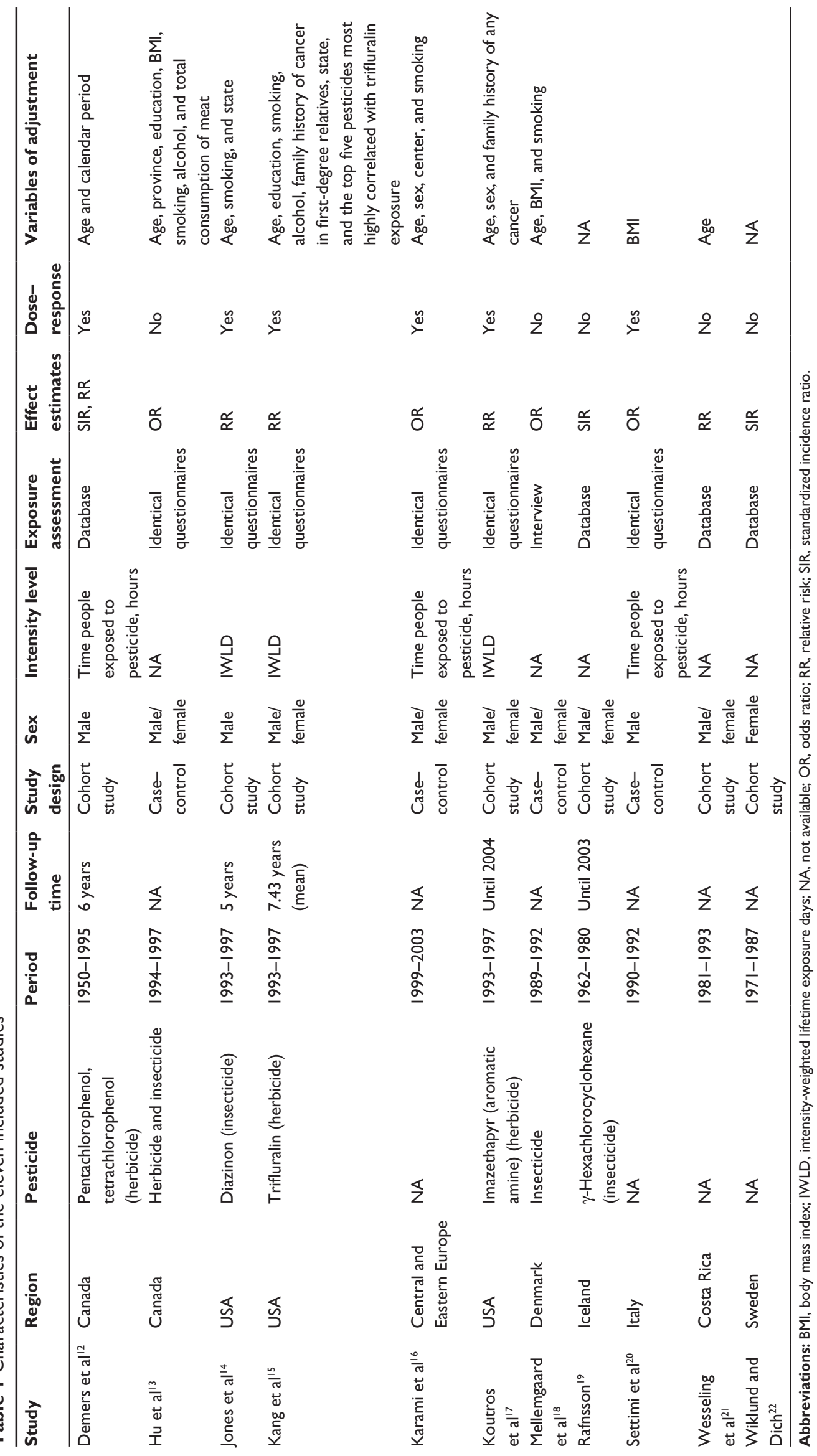




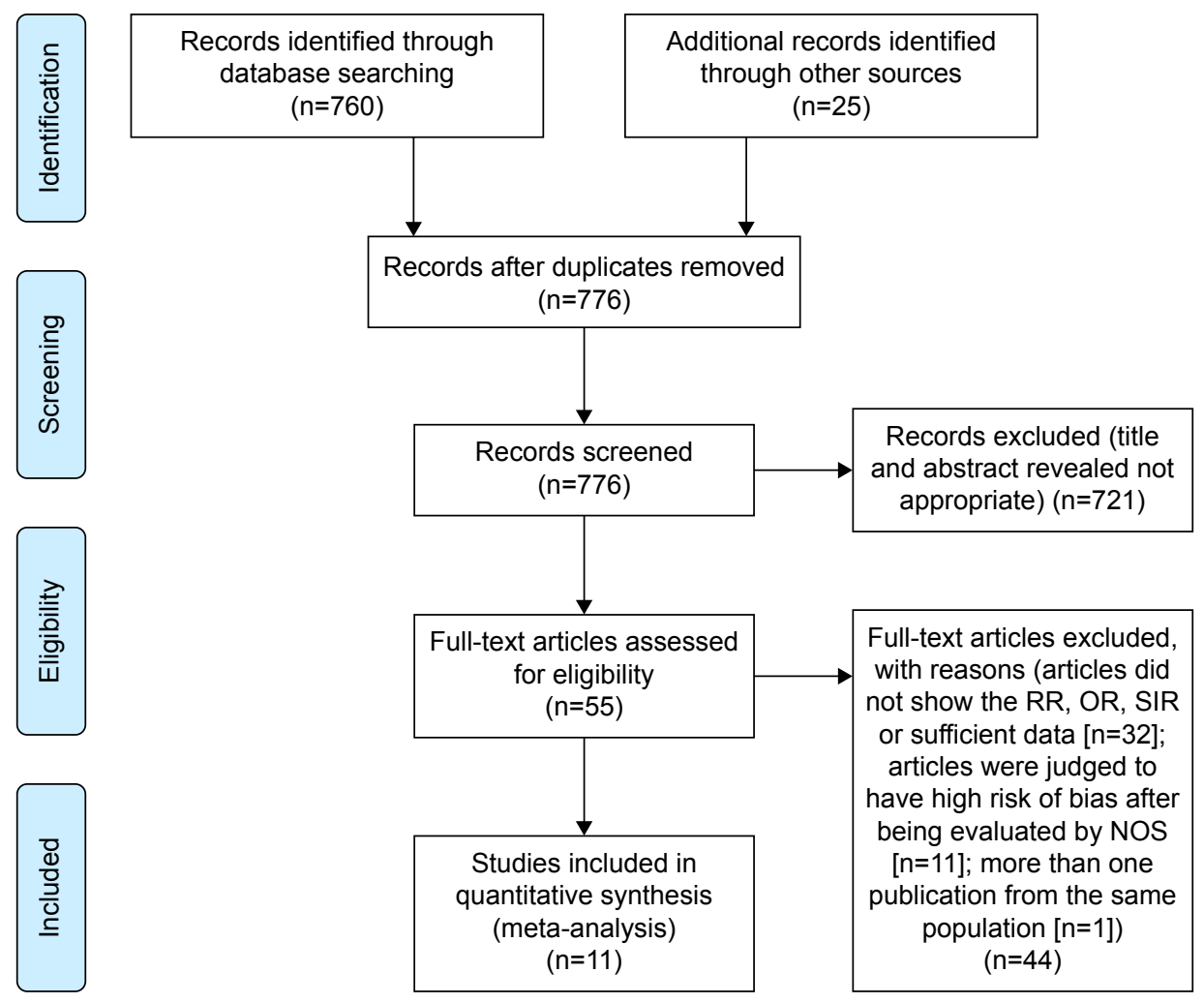

Figure I Preferred Reporting Items for Systematic reviews and Meta-Analyses (PRISMA) flow diagram showing an overview of the study selection process. Notes: From: Moher D, Liberati A, Tetzlaff J, Altman DG; The PRISMA Group. Preferred reporting items for systematic reviews and meta-analyses: the PRIMSA statement. PLoS Med. 2009;6(6):el000097. ${ }^{30}$

Abbreviations: NOS, Newcastle-Ottawa Assessment Scale; OR, odds ratio; RR, relative risk; SIR, standardized incidence ratio.

However, when we limited the studies to high quality articles (NOS score $>6$ ), the pooled RR estimated showed a significant association between pesticide exposure and kidney cancer (RR $=1.31,95 \%$ CI 1.12-1.51, $P<0.01$, random effects).

Next, we conducted a subgroup meta-analysis by various study characteristics (Table 3 ).

The subanalysis on ORs pointed to a positive association (pooled OR $=1.49,95 \%$ CI $1.23-1.8, P=0.14$ for heterogeneity; $I^{2}=33 \%$ ), although the subanalysis on RRs and SIRs did not reach formal significance.

When we limited studies to those with control for age or adjusted for more than two confounders, there was a statistically significant association between pesticide exposure and kidney cancer $(\mathrm{RR}=1.26,95 \% \mathrm{CI} 1.07-1.48, P=0.02$ for heterogeneity; $I^{2}=44 \%$ ).

In the analysis stratified by study design, a significant association between pesticide exposure and kidney cancer was found in case-control studies ( $\mathrm{RR}=1.49,95 \% \mathrm{CI}$ $1.23-1.8, P=0.14$ for heterogeneity; $\left.I^{2}=33 \%\right)$. Such a connection was not found in cohort studies ( $\mathrm{RR}=0.93,95 \% \mathrm{CI}$ $0.79-1.09, P=0.01$ for heterogeneity; $I^{2}=34 \%$ ).

We also investigated region, and a significant association between pesticide and risk of renal cancer was also observed in North America ( $R R=1.31,95 \%$ CI 1.09-1.59, random effects). However, no such finding was detected in Europe ( $R R=1.15,95 \%$ CI 0.82-1.6, random effects). Furthermore, when stratified by sex, there was no statistically significant correlation in either male or female $(\mathrm{RR}=1.26,95 \% \mathrm{CI}$ $0.94-1.69 ; \mathrm{RR}=1.01,95 \%$ CI $0.76-1.35$, respectively).

Significant association between pesticide exposure and renal cancer was observed in studies adjusted for more than two confounders $(\mathrm{RR}=1.34,95 \% \mathrm{CI} 1.13-1.6 ; P=0.08$ for heterogeneity; $I^{2}=34 \%$ ).

In the subgroup analysis by type of pesticide, there was no statistically significant association between insecticide exposure and increased renal cancer risk $(\mathrm{RR}=1.46$, $\left.I^{2}=50 \%\right)$. It is also observed in herbicide exposure ( $\left.\mathrm{RR}=1.22, P^{2}=78 \%\right)$.

\section{Evaluation of heterogeneity}

There was a significant heterogeneity among the included eleven studies $\left(I^{2}=69 \%, P<0.01\right)$. When we limited the studies to high quality studies (NOS score $>6$ ), there was moderate heterogeneity among them $\left(I^{2}=38 \%, P=0.05\right)$. These moderated quality studies may be the possible source of heterogeneity. 


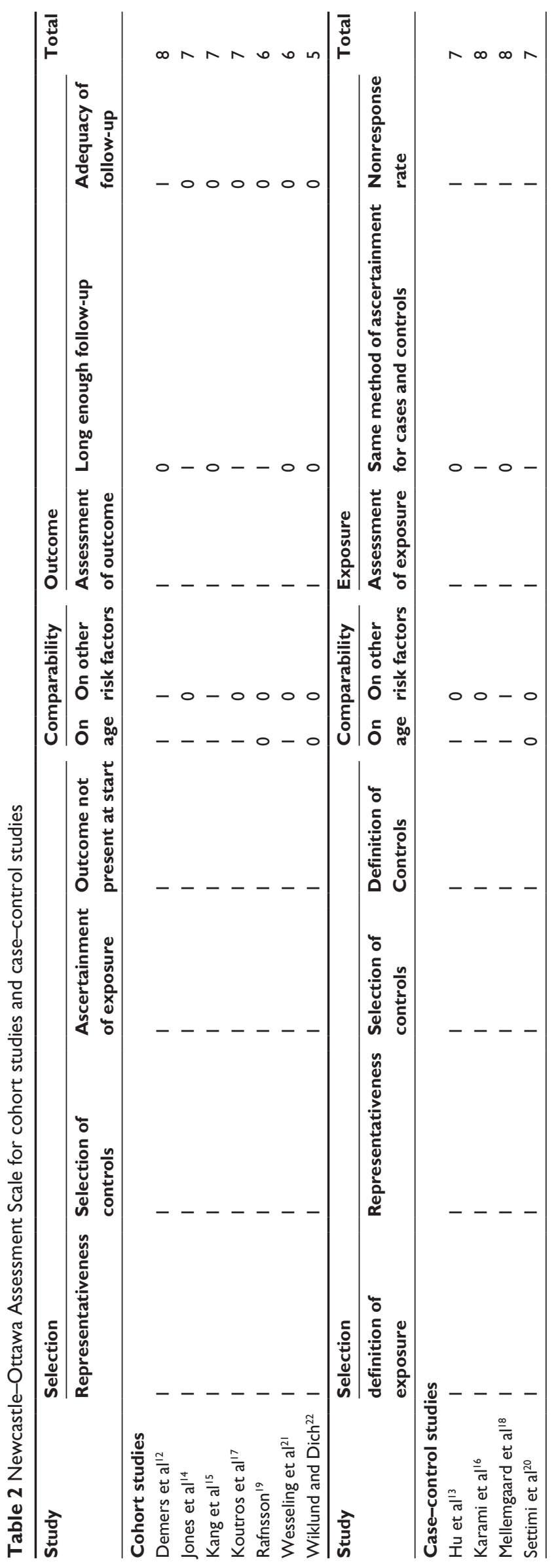

\section{Discussion}

Nowadays, the question whether pesticide exposure is independently associated with incidence of kidney cancer remains controversial. ${ }^{4,14,23}$ Our meta-analysis analyzed eleven epidemiologic studies, including seven cohort studies and four case-control studies, to evaluate the association between pesticide exposure and the risk of kidney cancer. Our findings indicated that pesticide exposure has a potential association of a $15 \%$ increased risk of kidney cancer. Especially when we limited to studies with high quality, the risk increased to $31 \%(P<0.01)$.

Results from our subgroup analysis showed the risk may relate to different adjustment for confounding factors, the type of effect estimates, study design, or the region of study population.

When we use studies with control for age or adjust for more than two confounders for subgroup analysis, it is more robust than reported in an overall analysis. It indicated that the association may be diluted by poor study methodologies. This is in keeping with the result of studies judged as high quality by NOS score. So pesticide exposure is probably an independent risk factor for kidney cancer.

In our subgroup analysis for case-control studies, a significant association between pesticide exposure and kidney cancer was found, which was not observed in cohort studies. Case-control studies require a control of matching factors associated with exposure rather than risk. Moreover, with a variable follow-up duration and censoring, a matched survival (time to event) analysis producing hazard ratios would have been a better strategy than reporting the $P$-values alone. ${ }^{24}$ So, case-control studies may be more potent than cohort studies in our study.

It tended to be more remarkable for studies with OR than that with SIR. In theory, RR should be calculated as the ratio of incidence in the exposed population to that in the unexposed. ${ }^{25}$ However, in some studies, owing to the lack of a nonexposure compared group, cancer incidence in exposure group was compared with incidence in the general population, which is known as SIR. The general population contains both exposed and unexposed groups, so articles using SIR to estimate the RR may underestimate the true RR. ${ }^{26}$

Our study has several limitations, which are listed as follows: 1) the sources of publication searched from databases were limited, and unpublished studies were not retrieved. 2) A major, unavoidable shortcoming pertained to the relatively short follow-up periods as in cancer research a follow-up time of $>10$ years is suitable. However, the follow-up 


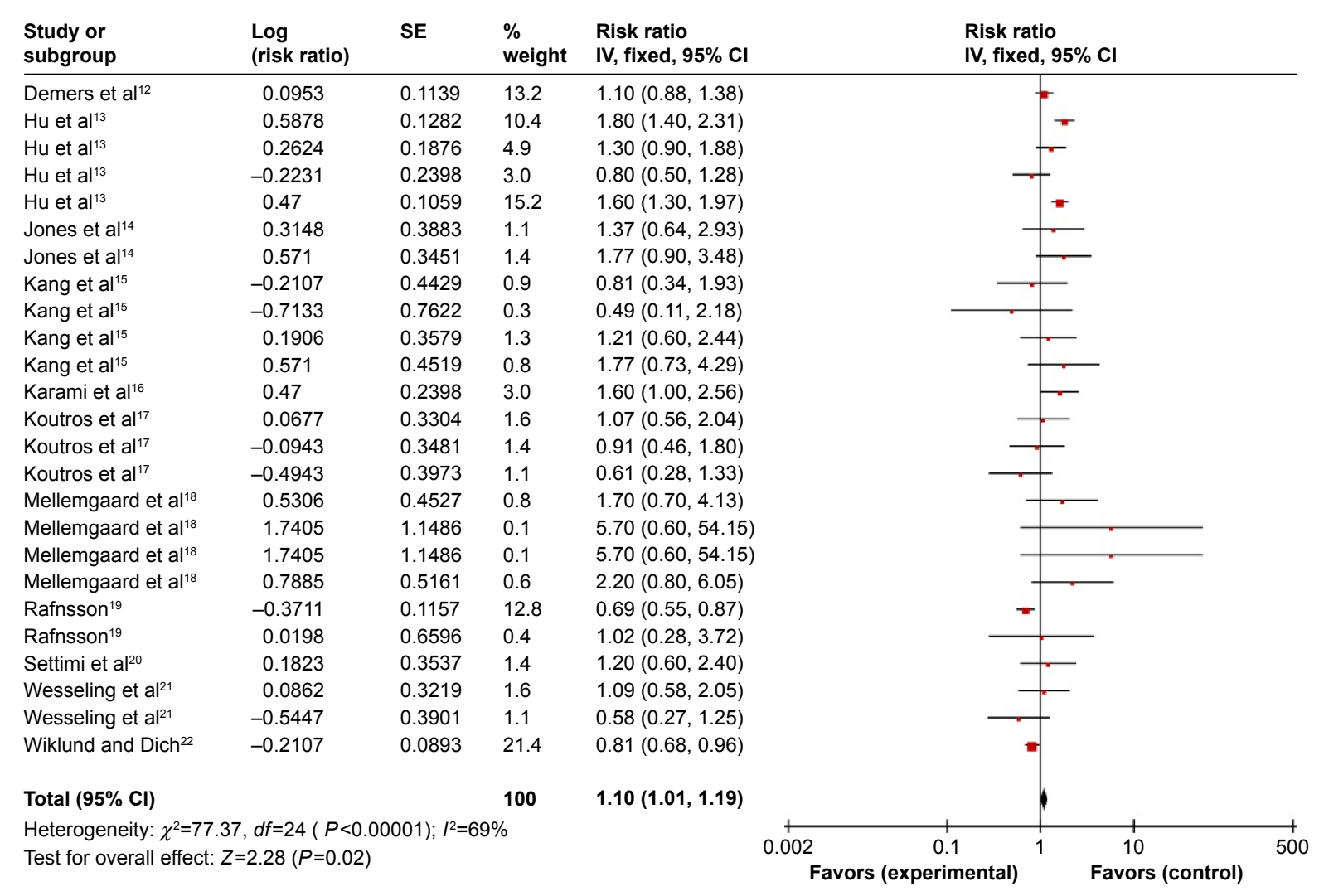

Figure 2 Forest plots depicting the risk estimates from included studies on the association between pesticide exposure and risk of kidney cancer. Abbreviations: $\mathrm{Cl}$, confidence interval; $d f$, degrees of freedom; IV, inverse variance; SE, standard error.

periods of the included cohort studies were $<10$ years. This may not be able to fully assess the relationship between exposure and outcome. 3) Both cohort and case-control studies were recruited in our study. Considering the existing heterogeneity, selecting a single global effect estimate to summarize the data might be inappropriate. So, the pooled estimates in our study should be treated with caution. Therefore,

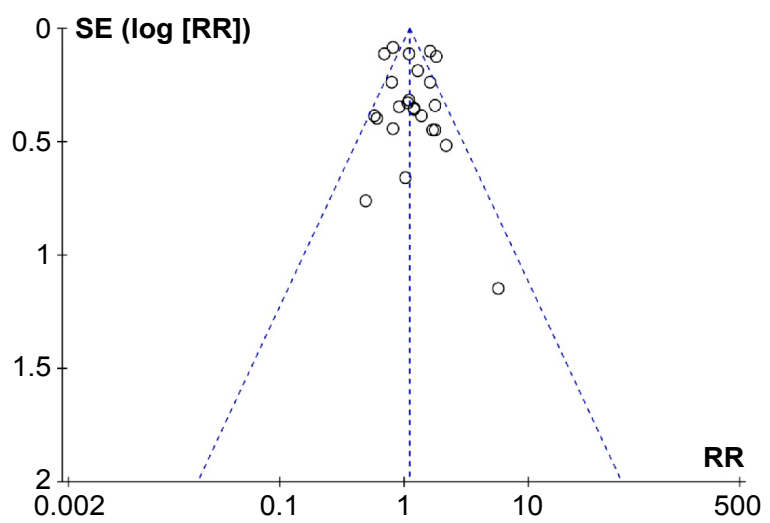

Figure 3 Funnel plots of overall analysis of relationship between pesticide exposure and kidney cancer.

Abbreviations: $\mathrm{RR}$, relative risk; SE, standard error. we conducted a subgroup analysis to explain the possible sources of heterogeneity. 4) Meta-analysis cannot solve the problem with confounding factors that could be internal in the recruited studies. Insufficient control of the known confounding factors could bring about a bias in a direction either toward exaggeration or underestimation of the risk estimates. ${ }^{27}$ In our study, the possibly insufficient control of confounding factors seemed to be a particular concern in the studies included: only six studies adjusted for three or more than three control factors. Therefore, potential confounding factors could not be completely excluded in the results of our meta-analysis. 5) Studies also indicated that pesticide exposure may be associated with the risk of precancerous lesions in animal research. ${ }^{28,29}$ However, in our study, we failed to discuss it. Further research is needed.

\section{Conclusion}

Our meta-analysis indicated that pesticide exposure was associated with the risk of kidney cancer. Further research should be conducted to confirm the findings in our study and better clarify the potential biological mechanisms. 
Table 3 Subgroup meta-analysis by various study characteristics

\begin{tabular}{|c|c|c|c|c|c|}
\hline \multirow[t]{2}{*}{ Subgroup } & \multirow{2}{*}{$\begin{array}{l}\text { Number } \\
\text { of studies }\end{array}$} & \multirow[t]{2}{*}{ RR (95\% Cl) } & \multirow[t]{2}{*}{$P$-value } & \multicolumn{2}{|c|}{ Heterogeneity } \\
\hline & & & & $P$-value & $I^{2}(\%)$ \\
\hline \multicolumn{6}{|c|}{ Type of pesticide } \\
\hline Herbicide & 4 & $1.22(0.96-1.54)$ & 0.1 & 0.05 & 50 \\
\hline Insecticide & 4 & $1.46(1.32-1.86)$ & 0.05 & $<0.01$ & 78 \\
\hline \multicolumn{6}{|l|}{ Study design } \\
\hline Cohort & 7 & $0.93(0.79-1.09)$ & 0.36 & 0.1 & 34 \\
\hline Case-control & 4 & $1.49(1.23-1.8)$ & $<0.01$ & 0.14 & 33 \\
\hline \multicolumn{6}{|l|}{ Sex } \\
\hline Male & 7 & $1.26(0.94-1.69)$ & 0.12 & $<0.01$ & 81 \\
\hline Female & 5 & I.0I (0.76-I.35) & 0.92 & 0.09 & 46 \\
\hline \multicolumn{6}{|l|}{ Region } \\
\hline Europe & 5 & I.I5 (0.82-I.6) & 0.42 & 0.01 & 57 \\
\hline North America & 4 & 1.31 (1.09-1.59) & $<0.01$ & 0.03 & 49 \\
\hline \multicolumn{6}{|c|}{ Effect estimates } \\
\hline $\mathrm{RR}$ & 4 & I.04 (0.83-I.3) & 0.74 & 0.44 & 0 \\
\hline OR & 4 & $1.49(1.23-1.8)$ & $<0.01$ & 0.14 & 33 \\
\hline SIR & 3 & $0.85(0.68-1.08)$ & 0.18 & 0.03 & 66 \\
\hline \multicolumn{6}{|c|}{ Exposure assessment } \\
\hline Questionnaires & 6 & $1.29(1.09-1.53)$ & $<0.01$ & 0.07 & 38 \\
\hline Database & 4 & $0.85(0.7-1.04)$ & 0.11 & 0.07 & 51 \\
\hline
\end{tabular}

Abbreviations: $\mathrm{Cl}$, confidence interval; $\mathrm{OR}$, odds ratio; RR, relative risk; SIR, standardized incidence ratio.

\section{Acknowledgments}

This study was supported by Zhejiang Province Key Project of Science and Technology (2014C04008-2), Health Sector Scientific Research Special Project (201002010), Scientific Research Foundation of The Ministry of Public Health (WKJ2012-2-009), and Natural Science Foundation of China (81372773).

\section{Disclosure}

The authors report no conflicts of interests in this work.

\section{References}

1. Torre LA, Bray F, Siegel RL, Ferlay J, Lortet-Tieulent J, Jemal A. Global cancer statistics, 2012. CA Cancer J Clin. 2015;65(2):87-108.

2. Bergstrom A, Hsieh CC, Lindblad P, Lu CM, Cook NR, Wolk A. Obesity and renal cell cancer - a quantitative review. Br J Cancer. 2001;85(7): 984-990.

3. Lindblad P. Epidemiology of renal cell carcinoma. Scand J Surg. 2004; 93(2):88-96.

4. Bassil KL, Vakil C, Sanborn M, Cole DC, Kaur JS, Kerr KJ. Cancer health effects of pesticides: systematic review. Can Fam Physician. 2007;53(10): 1704-1711.

5. Acquavella J, Olsen G, Cole P, et al. Cancer among farmers: a metaanalysis. Ann Epidemiol. 1998;8(1):64-74.

6. Ragin C, Davis-Reyes B, Tadesse H, et al. Farming, reported pesticide use, and prostate cancer. Am J Mens Health. 2013;7(2):102-109.

7. Dale KM, Coleman CI, Henyan NN, Kluger J, White CM. Statins and cancer risk: a meta-analysis. JAMA. 2006;295(1):74-80.

8. Van Maele-Fabry G, Libotte V, Willems J, Lison D. Review and metaanalysis of risk estimates for prostate cancer in pesticide manufacturing workers. Cancer Causes Control. 2006;17(4):353-373.
9. Greenland S. Quantitative methods in the review of epidemiologic literature. Epidemiol Rev. 1987;9:1-30.

10. Siristatidis C, Sergentanis TN, Kanavidis P, et al. Controlled ovarian hyperstimulation for IVF: impact on ovarian, endometrial and cervical cancer - a systematic review and meta-analysis. Hum Reprod Update. 2013;19(2):105-123.

11. Larsson SC, Mantzoros CS, Wolk A. Diabetes mellitus and risk of breast cancer: a meta-analysis. Int J Cancer. 2007;121(4):856-862.

12. Demers PA, Davies HW, Friesen MC, et al. Cancer and occupational exposure to pentachlorophenol and tetrachlorophenol (Canada). Cancer Causes Control. 2006;17(6):749-758.

13. Hu J, Mao Y, White K. Renal cell carcinoma and occupational exposure to chemicals in Canada. Occup Med. 2002;52(3):157-164.

14. Jones RR, Barone-Adesi F, Koutros S, et al. Incidence of solid tumours among pesticide applicators exposed to the organophosphate insecticide diazinon in the Agricultural Health Study: an updated analysis. Occup Environ Med. 2015;72(7):496-503.

15. Kang D, Park SK, Beane-Freeman L, et al. Cancer incidence among pesticide applicators exposed to trifluralin in the Agricultural Health Study. Environ Res. 2008;107(2):271-276.

16. Karami S, Boffetta P, Rothman N, et al. Renal cell carcinoma, occupational pesticide exposure and modification by glutathione S-transferase polymorphisms. Carcinogenesis. 2008;29(8):1567-1571.

17. Koutros S, Lynch CF, Ma X, et al. Heterocyclic aromatic amine pesticide use and human cancer risk: results from the U.S. Agricultural Health Study. Int J Cancer. 2009;124(5):1206-1212.

18. Mellemgaard A, Engholm G, McLaughlin JK, Olsen JH. Occupational risk factors for renal-cell carcinoma in Denmark. Scand J Work Environ Health. 1994;20(3):160-165.

19. Rafnsson V. Cancer incidence among farmers exposed to lindane while sheep dipping. Scand J Work Environ Health. 2006;32(3): 185-189.

20. Settimi L, Comba P, Bosia S, et al. Cancer risk among male farmers: a multi-site case-control study. Int J Occup Med Environ Health. 2001; 14(4):339-347. 
21. Wesseling C, Antich D, Hogstedt C, Rodriguez AC, Ahlbom A. Geographical differences of cancer incidence in Costa Rica in relation to environmental and occupational pesticide exposure. Int J Epidemiol. 1999; 28(3):365-374.

22. Wiklund K, Dich J. Cancer risks among female farmers in Sweden. Cancer Causes Control. 1994;5(5):449-457.

23. Payan-Renteria R, Garibay-Chavez G, Rangel-Ascencio R, et al. Effect of chronic pesticide exposure in farm workers of a Mexico community. Arch Environ Occup Health. 2012;67(1):22-30.

24. Ashutosh T, McGwin G. Case-control or cohort study? Br J Ophthalmol. 2011;95(8):1175.

25. Xu X, Wu J, Mao Y, et al. Diabetes mellitus and risk of bladder cancer: a meta-analysis of cohort studies. PLoS One. 2013;8(3):e58079.

26. Chaturvedi AK, Mbulaiteye SM, Engels EA. Underestimation of relative risks by standardized incidence ratios for AIDS-related cancers. Ann Epidemiol. 2008;18(3):230-234.
27. Larsson SC, Orsini N, Wolk A. Vitamin B6 and risk of colorectal cancer: a meta-analysis of prospective studies. JAMA. 2010;303(11):1077-1083.

28. Abdel-Daim M, El-Bialy BE, Rahman HG, Radi AM, Hefny HA, Hassan AM. Antagonistic effects of Spirulina platensis against subacute deltamethrin toxicity in mice: Biochemical and histopathological studies. Biomed Pharmacother. 2016;77:79-85.

29. Abdel-Daim MM. Synergistic protective role of ceftriaxone and ascorbic acid against subacute diazinon-induced nephrotoxicity in rats. Cytotechnology. 2016;68(2):279-289.

30. Moher D, Liberati A, Tetzlaff J, Altman DG. Preferred reporting items for systematic reviews and meta-analyses: the PRISMA statement. PLoS Med. 2009;6(7):e1000097.
OncoTargets and Therapy

\section{Publish your work in this journal}

OncoTargets and Therapy is an international, peer-reviewed, open access journal focusing on the pathological basis of all cancers, potential targets for therapy and treatment protocols employed to improve the management of cancer patients. The journal also focuses on the impact of management programs and new therapeutic agents and protocols on

\section{Dovepress}

patient perspectives such as quality of life, adherence and satisfaction. The manuscript management system is completely online and includes a very quick and fair peer-review system, which is all easy to use. Visit http://www.dovepress.com/testimonials.php to read real quotes from published authors. 